\section{Peeking inside AMD}

\section{By Benjamin Boettner, Associate Editor}

Conflicting results from two 2012 studies made it unclear whether IL-18 was a cause of or a potential therapy for age-related macular degeneration. ${ }^{1,2}$ A tiebreaker study suggests the latter, showing that the cytokine was at least as effective as a VEGF inhibitor in mouse models of wet age-related macular degeneration. ${ }^{3}$

Age-related macular degeneration (AMD) is the leading cause of blindness in people older than 65 and affects 30-50 million people worldwide. In about $10 \%$ of patients, the disease progresses to the wet form, which is characterized by choroidal neovascularization (CNV) - newly sprouting blood vessels originating from the outer vascular layer of the eye.

These vessels break through the retinal pigment epithelium (RPE), where they hemorrhage. The resulting blood clots damage the RPE, leaving photoreceptors unprotected and causing them to degenerate. ${ }^{4}$ Wet AMD typically is treated with intravitreal injections of molecules that inhibit VEGF.

In 2012, a team at Trinity College Dublin led by Matthew Campbell and Sarah Doyle found a new target in the disease. The team reported a protective role for the Nlr family pyrin domain containing 3 (Nlrp3; Nalp3; Cias1) inflammasome and its downstream effector Il-18. ${ }^{1}$

Campbell is a research assistant professor at the Smurfit Institute of Genetics at Trinity College Dublin. Doyle is an assistant professor at the Trinity College Dublin School of Medicine.

That same year, however, researchers at the University of Kentucky reported that intravitreal injection of recombinant human IL-18 provoked an AMD phenotype in mice. ${ }^{2}$

The newest study from Campbell and Doyle supports their original findings. ${ }^{3}$

In a mouse model of wet AMD, the group intravitreally injected recombinant human IL-18 alone or in combination with a murine Vegf-neutralizing antibody. IL-18 by itself reduced the severity of $\mathrm{CNV}$ at least as efficiently as the anti-VEGF antibody. The combination showed additive effects.

Moreover, the researchers showed that systemically increasing levels of Il-18 also protected mice against CNV. Systemic Il-18 treatment for two weeks before induction of CNV suppressed neovascularization and boosted the effects of the intravitreally injected anti-Vegf antibody. The team did not directly compare systemic Il-18 with intravitreal anti-Vegf therapy.

Results were published in Science Translational Medicine. The study was coauthored by researchers from the Royal Victoria Eye and Ear Hospital, the National Eye Institute of the NIH and the Ophthiris
Discovery Performance Unit of GlaxoSmithKline plc, which provided the recombinant human IL-18.

"The paper describes a somewhat counterintuitive approach for the treatment of CNV secondary to wet AMD-using a proinflammatory cytokine, namely IL-18, to dampen later stage VEGF-mediated choroidal vessel angiogenesis," said Kay Rittenhouse, senior director of ophthalmology and external R\&D innovation at Pfizer Inc.

Mechanistically, Campbell thinks that "it is highly likely that IL-18 is acting directly on endothelial cells when injected intravitreally. However, when administered systemically, IL-18 is likely inducing specific subpopulations of immune cells-NK cells and activated T cells-that have antiangiogenic potency." Studies to determine the actual mechanism are under way in Campbell's laboratory.

According to Rittenhouse, it will be important to determine "whether the mechanism by which IL-18 dampens neovascularization is due to its proinflammatory activity."

\section{Envisioning the IL-18 path}

According to Rittenhouse and Campbell, the contradictory results from the Trinity team and the University of Kentucky group could be explained by the different doses of IL-18 used in the studies.

The exact IL-18 concentrations were not specified in the 2012 report by the Kentucky team. Based on titration curves performed by the Trinity team, Campbell said that the other group almost certainly used doses of IL-18 that were above a therapeutic and physiological threshold and could have damaged the RPE.

Doyle noted that the Trinity team also saw adverse effects on the RPE and other retinal cells at extremely high doses of IL- 18 .

Alternatively, Rittenhouse told SciBX that "recombinant IL-18s may differ structurally from one another in important ways that link to responses. These structural characteristics need to be highlighted to minimize potential adverse effects."

With the strengthened case for using IL-18 to treat AMD, Lois Smith, a professor of ophthalmology at Harvard Medical School and the Boston Children's Hospital, thinks that the next step should be the clinic.

She said that animal models of AMD have multiple shortcomings and noted that inflammatory and gene expression changes in response to burns or injury as occurs in laser-induced CNV differ markedly between mice and humans. 5

"It could be possible to test IL-18 in a small pilot study in human subjects with advanced AMD that already have lost vision but whose retinas still allow observations of effects on the neovasculature," said Smith.

Campbell said that Trinity College and its clinical partners are designing a trial of IL-18 in patients with wet AMD.

"Although IL-18 alone appears to contain CNV as well as antiVEGF treatment, it is likely that, to begin with, a combined therapy might be most acceptable," said Doyle. She added that the Trinity 


\section{ANALYSIS}

\section{TARGETS \& MECHANISMS}

team is conducting preclinical studies to further define the safety and mechanism of action of IL-18 treatment.

Trinity has filed for a patent covering the use of recombinant human IL-18 to treat CNV secondary to wet AMD. GlaxoSmithKline has an option to license the IP.

Boettner, B. SciBX 7(17); doi:10.1038/scibx.2014.476

Published online May 1, 2014

\section{REFERENCES}

1. Doyle, S.L. et al. Nat. Med. 18, 791-798 (2012)

2. Tarallo, V. et al. Cell 149, 847-859 (2012)

3. Doyle, S.L. et al. Sci. Transl. Med.; published online April 2, 2014; doi:10.1126/scitransImed.3007616

Contact: Matthew Campbell, Smurfit Institute of Genetics at Trinity College Dublin, Dublin, Ireland

e-mail: matthew.campbell@tcd.ie
4. Jager, R.D. et al. N. Engl. J. Med. 358, 2606-2617 (2008)

5. Seok, J. et al. Proc. Natl. Acad. Sci. USA 110, 3507-3512 (2013)

COMPANIES AND INSTITUTIONS MENTIONED

Boston Children's Hospital, Boston, Mass.

GlaxoSmithKline plc (LSE:GSK; NYSE:GSK), London, U.K.

Harvard Medical School, Boston, Mass.

National Eye Institute, Bethesda, Md.

National Institutes of Health, Bethesda, Md.

Pfizer Inc. (NYSE:PFE), New York, N.Y.

Royal Victoria Eye and Ear Hospital, Dublin, Ireland Smurfit Institute of Genetics at Trinity College Dublin,

Dublin, Ireland

Trinity College Dublin, Dublin, Ireland

Trinity College Dublin School of Medicine, Dublin, Ireland University of Kentucky, Lexington, Ky. 